\title{
Applications of High-Resolution Aberration-Corrected STEM Imaging to Studies of the Behavior of Nanophase Materials at Elevated Temperatures
}

\author{
Lawrence F. Allard,* Karren L. More* and Jingyue (Jimmy) Liu \\ * Materials Science and Technology Div., Oak Ridge National Laboratory, Oak Ridge, TN 37831 \\ ** Center for Nanoscience and the Department of Physics \& Astronomy, University of Missouri-St. \\ Louis, St. Louis, MO 63121
}

Electron microscopes equipped with aberration correctors that can provide image resolution in the sub-Ångström regime have moved from the theory and promise of a decade ago when the first prototype instruments were demonstrated, to nearly routine, common use today, with more than 70 instruments currently in the field. A significant majority of today's instruments have aberration correctors for the probe-forming optics, because of the sensitivity of high-angle annular dark-field (HA-ADF) STEM images atomic number differences. For example, heavy metal atoms in catalytic materials are readily imaged in HA-ADF mode, whereas the high phase contrast information in conventional bright-field images (even with aberration correction) often completely obscures the information from single atoms or small clusters. Bright-field images, however, are less sensitive to sample orientation, so that lattice fringes are often more readily seen. STEM imaging allows both HA-ADF and BF images to be acquired simultaneously, at Angström-level resolution, so the lattice periodicities seen in the BF images can be correlated to the DF information. The addition of energyloss and energy-dispersive spectrometers, and a new generation of specialized specimen holders with stable heating and gas-reaction technologies, extends the capability of the aberration-corrected microscope to new levels of materials characterization performance. Examples of the characterization of a variety of nanophase materials using a JEOL 2200FS STEM/TEM instrument equipped with a CEOS $\mathrm{GmbH}$ probe corrector, an in-column Omega energy filter, a Bruker Co. silicon-drift x-ray detector, and specimen heating and gas-reaction holders utilizing Protochips Aduro $^{\mathrm{TM}}$ heating technology will be presented in this talk; selected examples are given below.

An example of the benefits of aberration-corrected STEM imaging of catalytic materials is shown in Figs. 1 and 2, which illustrate the behavior of Pt species on a carbon support (e.g. for fuel cell catalyst studies), heated in-situ. Figures $1 \mathrm{a}$ and $1 \mathrm{~b}$ are, respectively, simultaneously acquired HA$\mathrm{ADF}$ and $\mathrm{BF}$ images from a thin area of the carbon support; the support surface is nearly planar since the imaged clusters are essentially at the same focus level. Both amorphous clusters and mononuclear species of Pt are seen in the high contrast ADF image with only one cluster apparently roughly organized into a nanocrystal (arrow). This is confirmed by comparison to the BF image, and illustrates the benefit of acquiring such image pairs (not possible with TEM imaging modes). Figure 2 is a sequence of HA-ADF images from a "movie" constructed from a dozen images of the same sample area held for varying times at a nominal $150^{\circ} \mathrm{C}$. The electron beam was blanked between each heating cycle, which comprised 8 images at $90 \mathrm{sec}$ each, 3 at $12 \mathrm{~min}$ each, and 1 at 24min. These images illustrate the low-temperature behavior of Pt on carbon: Pt particles do not dissolve and move atomically across the carbon surface to deposit on nearby particles; individual (initially amorphous) clusters move instead across the surface and coalesce with nearby clusters, forming larger crystalline nanoparticles. This differentiates traditional Ostwald ripening vs a coalescence mechanism for particle growth in this system.

The formation of $\mathrm{PdZn}$ alloy nanoparticles has been proposed to explain the catalytic performance of $\mathrm{Pd} / \mathrm{ZnO}$ catalysts [1]. The detailed structure and the formation processes of the $\mathrm{PdZn}$ alloy nanoparticles, however, are not well understood. We used well-defined $\mathrm{ZnO}$ nanoribbons as the support material to study the evolution of Pd during in situ heating experiments. Sub-Ångström imaging of both the support and the Pd nanoparticles provided insights into the growth processes of $\mathrm{Pd}$ nanoparticles and the interaction of $\mathrm{Pd}$ nanoparticles with the $\mathrm{ZnO}$ support. When the $\mathrm{Pd} / \mathrm{ZnO}$ 
catalyst precursor material was heated to $400^{\circ} \mathrm{C}$ for $55 \mathrm{~min}$, a novel reconstructed surface structure formed on Pd-Zn nanoparticles (Fig. 3a). After heating at $500^{\circ} \mathrm{C}$ for $30 \mathrm{~min}, \mathrm{PdZn}$ alloy nanoparticles grew epitactically with the $\mathrm{ZnO}(0001)$ surface, with well defined $\{111\}$ faces exposed (Fig. 3b). These observations may help explain the unique activity/selectivity data reported in the literature [2].

\section{References}

[1] N. Iwasaa, S. Masudaa, N. Ogawaa and N. Takezawa, Applied Catalysis A: General, 125 (1995) 145.

[2] This research at the Oak Ridge National Laboratory's High Temperature Materials Laboratory was sponsored in part by the U. S. Department of Energy, Office of Energy Efficiency and Renewable Energy, Vehicle Technologies Program, and by the University of Missouri-St. Louis.
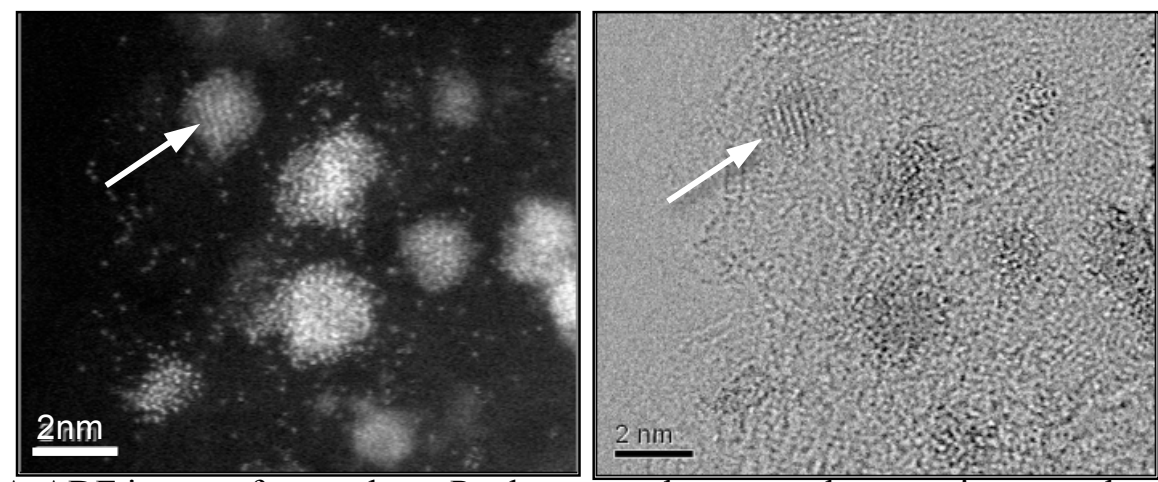

Fig. 1. a) HA-ADF image of amorphous Pt clusters and mononuclear species on carbon support; pseudocrystal is arrowed. b) corresponding BF image; lattice in particle also seen, but no mononuclear species.
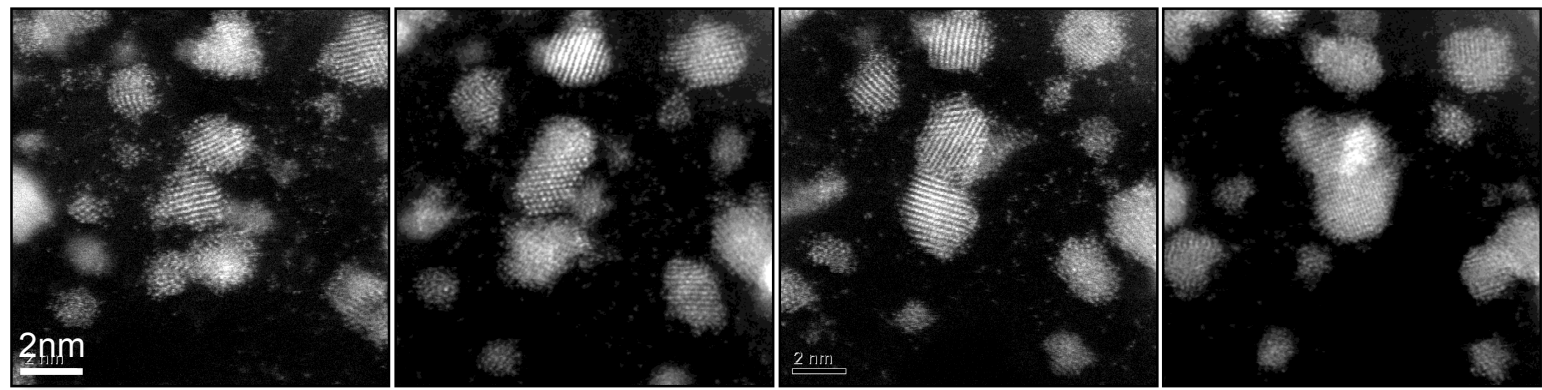

Fig. 2. Movie sequence of $\mathrm{Pt} / \mathrm{C}$ at a nominal $150^{\circ} \mathrm{C}$, showing growth of nanocrystals largely by a coalescence mechanism; see text for details.
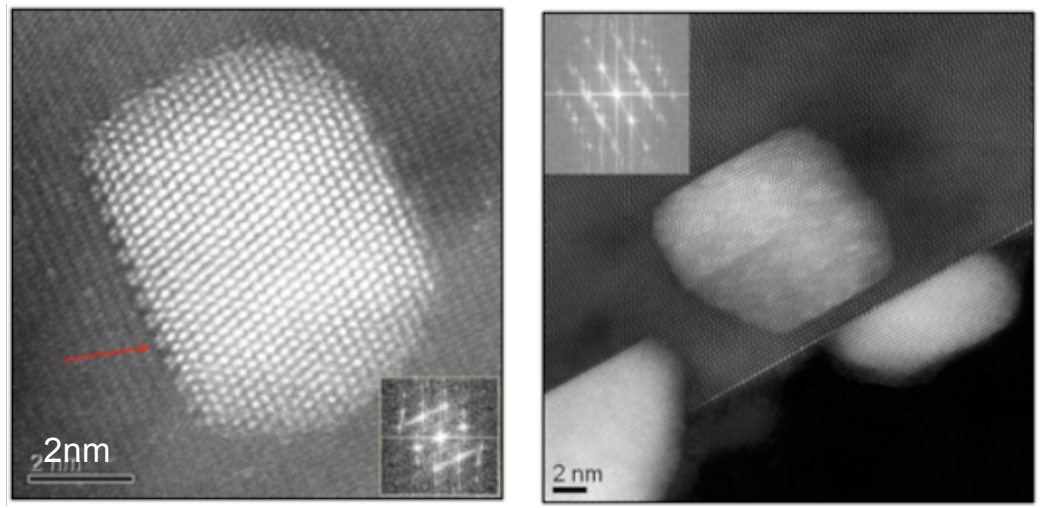

Fig. 3. a) $\mathrm{Pd}-\mathrm{Zn}$ nanocrystal grown at $400^{\circ} \mathrm{C}$ on $\mathrm{ZnO}$ nanoribbon surface, showing unique reconstructed surface (arrow); b) after time at $500^{\circ} \mathrm{C} \mathrm{PdZn} \mathrm{particles} \mathrm{grew} \mathrm{epitactically,} \mathrm{with}\{111\}$ facets. 